\title{
Functional characterization of RNase H1 proteins in Arabidopsis thaliana
}

Jan Kuciński ${ }^{1}$, Aleksandra Kmera ${ }^{2,3}$, M. Jordan Rowley ${ }^{1,4}$, Pragya Khurana ${ }^{1}$, Marcin Nowotny ${ }^{2}$, Andrzej T. Wierzbicki ${ }^{1,2^{*}}$

${ }^{1}$ University of Michigan, Department of Molecular, Cellular and Developmental Biology, Ann Arbor, MI, USA

${ }^{2}$ Laboratory of Protein Structure, International Institute of Molecular and Cell Biology, Warsaw, Poland

${ }^{3}$ Current address: Department of Protein Biosynthesis, Institute of Biochemistry and Biophysics, Warsaw, Poland

${ }^{4}$ Current address: Department of Biology, Emory University, Atlanta, GA, USA

*Corrersponding author, wierzbic@umich.edu 


\begin{abstract}
RNase H1 is an endonuclease specific towards RNA:DNA hybrids. Members of this protein family are present in most living organisms and are essential for removing RNA that base pairs with DNA. It prevents detrimental effects of RNA:DNA hybrids and is involved in several biological processes. We show that Arabidopsis thaliana contains four RNase H1-like proteins originating from two gene duplication events and alternative splicing. These proteins have the canonical RNase $\mathrm{H} 1$ activity, which requires at least four ribonucleotides for activity. Two of those proteins are nuclear, one is localized to mitochondria and one to plastids. While the nuclear RNases H1 are dispensable, the presence of at least one organellar RNase H1 is required for embryonic development. The plastid protein RNH1C affects plastid DNA copy number and sensitivity to hydroxyurea. This indicates that three genomes present in each plant cell are served by at least one specialized RNase H1 protein.
\end{abstract}

\title{
INTRODUCTION
}

Double stranded nucleic acids, which contain deoxyribonucleotides on one strand and one or more ribonucleotides on the other strand are known as RNA:DNA hybrids. They are common byproducts of replication, transcription and other processes. Ribonucleotides within RNA:DNA hybrids are specifically removed by a class of endonucleases known as RNases $\mathrm{H}^{1}$. RNases $\mathrm{H} 2$ are multi subunit complexes capable of removing even individual ribonucleotides incorporated in double stranded DNA and have been studied in various eukaryotes, including plants ${ }^{2,3}$. RNases $\mathrm{H} 1$ act as monomers ${ }^{4}$ and require at least four ribonucleotide incorporated into double stranded DNA to bind the substrate 5 .

Among the substrates of RNases H1 are R-loops ${ }^{6}$ (RNA:DNA hybrid and a displaced ssDNA strand) which are often formed during transcription and replication ${ }^{7-9}$. These structures have also been implicated in DNA repair ${ }^{10-12}$, telomere maintenance ${ }^{13}$, IgG classswitch recombination ${ }^{14}$ and regulation of gene expression ${ }^{9,15}$. RNase H1 digests ribonucleotides within its substrate in a metal ion-dependent manner ${ }^{16}$, leading to single stranded DNA formation. Main feature of the proteins encoded by RNase H1 genes is the presence of the catalytic domain ${ }^{16,17}$. Additionally, some bacterial and most eukaryotic RNase H1 proteins contain a RNA:DNA hybrid binding domain $(\mathrm{HBD})^{18}$.

Genes encoding RNase $\mathrm{H} 1$ proteins are present in the vast majority of living organisms, including Archea ${ }^{19,20}$, Bacteria ${ }^{21}$ and all kingdoms of Eukarya ${ }^{1}$. They are not essential in prokaryotes and lower eukaryotes but are required for survival in higher eukaryotes $^{1}$. Eukaryotic genomes usually contain unique RNase H1 genes ${ }^{1,21}$, which may 
however be subject to alternative splicing ${ }^{22}$. Arabidopsis thaliana RNases H1 are encoded by three different genes with different predicted subcellular localizations ${ }^{23}$.

Among the three RNase H1 proteins in Arabidopsis thaliana, only the chloroplastlocalized paralog has been studied so far ${ }^{23}$. It is essential for proper plastid development by maintaining the integrity of chloroplast DNA. It works with its interacting partner, DNA gyrase, to resolve transcription-replication conflicts and prevent DNA damage ${ }^{23}$. The role of the remaining two proteins remains unknown beyond the presumption that they resolve Rloops, which are relatively common in the Arabidopsis genome ${ }^{24}$.

Here, we characterize all RNase $\mathrm{H} 1$ proteins detectable in the Arabidopsis genome. We identify two ancient gene duplication events, which led to the formation of RNase H1 proteins targeted to various cellular compartments in monocots and dicots. A more recent duplication and alternative splicing produced four RNase H1 proteins in Arabidopsis thaliana, two of which are targeted to the nucleus, one to the chloroplast and one to the mitochondria. The proteins localized to endosymbiotic organelles are required for proper embryonic development. These proteins have the canonical RNase $\mathrm{H} 1$ activity and are involved in nucleic acid metabolism.

\section{RESULTS}

\section{Origin of angiosperm RNases $\mathrm{H1}$}

Plant genomes have been shown to encode multiple RNase H1-proteins with different subcellular localizations ${ }^{23}$. To determine their evolutionary origins, we performed an in-depth phylogenetic analysis of plant RNase H1-like proteins. We first used BLAST to identify all plant proteins, which display sequence similarity to Arabidopsis thaliana AtRNH1C ${ }^{23}$ and contain both RNase H1 and RNA:DNA hybrid binding (HBD) domains. The list of identified proteins is in the Supplemental File 1. The identified proteins were subject to a simultaneous Bayesian alignment and phylogenetic analysis implemented using BAli-Phy package in order to integrate over the uncertainty in both the phylogeny and alignment ${ }^{25}$. In parallel, we predicted the subcellular localization of each identified protein using the TargetP prediction tool $^{26}$. RNase H1-like proteins from angiosperms grouped into four distinct clades (Fig. 1, Fig. S1). The first group included proteins from monocots with mostly nuclear predicted localization. The second group included proteins from monocots with mostly chloroplast or mitochondrial predicted localization (referred to as organellar). The third group included proteins from dicots with mostly organellar localization. Finally, the fourth group included 
proteins from dicots with nuclear localization (Fig 1, Fig. S1, Fig. 2A). Phylogenetic relationships between these proteins indicate that the common ancestor of monocots and dicots had one RNase H1-like protein. Two independent gene duplication events then led to both monocots and dicots acquiring at least two proteins with distinct subcellular localizations.

\section{Origin of four RNases $\mathrm{H1}$ in Arabidopsis}

Arabidopsis thaliana has been shown to contain three genes encoding RNase H1-like proteins: RNH1A (AT3G01410), RNH1B (AT5G51080) and RNH1C (AT1G24090). Products of these genes localize to the nucleus, mitochondria and chloroplasts, respectively ${ }^{23}$. While the split into nuclear and organellar proteins occurred early in dicot evolution (Fig. 1), the origin of two organellar proteins remains unknown. To determine the relationship between RNH1B and RNH1C we analyzed the phylogeny of RNase H1-like proteins in Brasicaceae (Fig. 2B). The phylogenetic tree identified one clade of nuclear and two distinct clades of organellar RNase H1-like proteins (Fig. 2B). This indicates that a second diversification event occurred in the common ancestor of Brasicaceae, which led to the formation of two organellar proteins. While in Arabidopsis thaliana these proteins are localized to mitochondria and chloroplasts, these specific localizations cannot be conclusively predicted for other Brasicaceae.

Multiple subcellular localizations of proteins produced from unique RNase H1 genes in animals are commonly determined by alternative splicing ${ }^{27}$. Araport1 1 genome annotation $^{28}$ suggest a similar mechanism in Arabidopsis thaliana, where three splice variants of RNH1B (AT5G51080) have been identified (Fig. 2C). We confirmed these annotations using 5'RACE (Fig. 2D). While one splice variant (AT5G51080.1 and AT5G51080.2) encodes a full-length protein, the second variant (AT5G51080.3) has a truncated mitochondrial presequence region and is predicted to localize to the nucleus (Fig. 2E). We conclude that Arabidopsis thaliana contains four RNase H1-like proteins originating from two independent gene duplication events and alternative splicing.

\section{RNase H1-like proteins from Arabidopsis have canonical RNase H1 activity}

Although Arabidopsis thaliana RNase H1-like proteins have extensive sequence similarity with RNase $\mathrm{H} 1$ proteins, their exact enzymatic activity remains unknown. RNH1C has been shown to remove binding sites of S9.6 antibody from chloroplast DNA ${ }^{23}$. This however, does not conclusively show RNase H1 activity of this protein. To determine if 
RNH1A and RNH1B are indeed RNases H1, we incubated the recombinant proteins with oligonucleotide substrates. Double stranded DNA and RNA was not digested by the recombinant proteins (Fig. 3AB). The same was true for oligonucleotides containing one or two ribonucleotides, which differentiates RNase H1 from RNase H2. Oligonucleotides containing four ribonucleotides were however digested by both RNH1A and RNH1B (Fig. $3 \mathrm{AB}$ ). We did not test RNH1C because we were unable to produce a soluble recombinant protein. These results indicate that RNH1A and RNH1B have the canonical RNase H1 activity. Because of the extensive sequence similarity, especially within the catalytic domain, RNH1C is likely to have the same enzymatic activity.

\section{RNH1A and RNH1B do not affect development}

RNH1C has been shown to be required for proper chloroplast development ${ }^{23}$. To determine the roles of all three RNase H1 encoding genes, we obtained T-DNA mutants in RNH1A (SALK_150285C) and RNH1B (SAIL_1174_C11) as well as the previously published RNH1C (SAIL_97_E11). Single mutants atrnh1a and atrnh1b did not exhibit any obvious developmental phenotypes (Fig. 4A-C). atrnh1c mutant had the expected pale leaf and dwarf phenotype (Fig. 4D). The pale green phenotype of atrnhlc single mutant was confirmed by chlorophyll content quantification (Fig. 4I).

To determine if the studied T-DNA mutants may express truncated proteins, we performed RT-PCR with primers upstream or downstream of the T-DNA insertion sites (Fig. $4 \mathrm{~J})$. All mutants had strongly and significantly reduced RNA accumulation downstream of TDNA insertions (Fig. 4KL). This indicates that these mutants are unlikely to produce truncated proteins containing the $\mathrm{C}$-terminal RNase $\mathrm{H} 1$ domain. RNA accumulation upstream of T-DNA was reduced in atrnhla, unchanged in atrnhlc and strongly increased in atrnhlb (Fig. 4KL). This indicates that a truncated N-terminal fragment is unlikely to be produced in atrnhla. Truncated N-terminal fragments may be produced in atrnhlb and atrnhlc, however atrnhlb is not expected to contain full length RNase $\mathrm{H} 1$ domain. On the other hand, atrnhlc may produce a truncated protein including the RNase $\mathrm{H}$ domain (Fig. 4J), which indicates that the C-terminal part of the protein is important for its function. The strong increase of upstream RNA accumulation in atrnh $1 b$ may indicate the presence of an autoregulatory mechanism within $R N H 1 B$.

\section{Presence of both RNH1B and RNH1C is required for viability}


Because RNases $\mathrm{H} 1$ are required for viability in animals ${ }^{27}$, we tested the phenotypes of all combinations of RNH1 double mutants. The atrnhla, atrnhlb double mutant did not show any visible developmental phenotypes (Fig. 4E) and the phenotype of the atrnhla, atrnhlc double mutant was similar to the atrnhlc single mutant. Phenotypic similarity of atrnhlc single mutant and atrnhla, atrnhlc double mutant was confirmed by chlorophyll content quantification (Fig. 4I). Despite several attempts we were unable to obtain the atrnhlb, atrnh1c double mutant. Among $211 \mathrm{~F} 1$ plants obtained from crossing $a t r n h 1 b^{-/}$with atrnh $1 c^{+/-}$we found no viable double homozygous mutant. However, the cross resulted in about $25 \%$ of seeds not developing properly (Fig. 5A-C), which likely are the double mutants. These improperly developing seeds contain aborted embryos which stop developing at approximately heart-shape stage of embryonic development (Fig. 5DE). These results suggest that while the nuclear RNH1A is not required for viability, the presence of at least one organellar protein RNH1B or RNH1C is essential.

\section{RNH1C is involved in chloroplast nucleic acid metabolism}

RNH1C has been shown to be involved in chloroplast DNA maintenance ${ }^{23}$. To test if RNases H1 expressed from the three Arabidopsis genes affect DNA copy number in various cellular compartments we performed real time PCR with primers specific to nuclear, mitochondrial and chloroplast genomes. atrnhlc mutant and atrnhla, atrnhlc double mutant contained approximately three times more chloroplast DNA than wild type mutant plants (Fig. $6 \mathrm{~A}-\mathrm{C})$. This result indicates that RNH1C protein is involved in controlling DNA copy number in chloroplasts, which is consistent with its postulated role in chloroplast genome maintenance ${ }^{23}$. Surprisingly, atrnhla, atrnhlb mutants and the atrnhla, atrnhlb double mutant did not affect the relative content of the three genomes (Fig. 6A-C). This indicates that nuclear and mitochondrial RNases H1 have different roles than the chloroplast RNase H1. Alternatively, some or all RNase H1 proteins may be targeted to more than one compartment.

The increased amount of chloroplast DNA in atrnhlc mutants might be a result of impaired DNA replication. To test this possibility, we treated wild type plants and RNase H1 mutants with replication stress and measured root length. To induce replication stress, we applied hydroxyurea ${ }^{2,3,29}$, which is known to inhibit replication by inhibiting rNTP reductase and therefore decreasing the amount of available dNTPs ${ }^{30}$. Treatment with $2 \mathrm{mM}$ hydroxyurea caused a decrease of root length in wild type plants to approximately $40 \%$ of root length in untreated plants (Fig. 6 E). rnhla and $r n h 1 b$ mutants had no effect on hydroxyurea sensitivity (Fig. 6 E). However, roots of atrnhlc mutant and atrnhla, atrnhlc double mutant grew to less 
than $30 \%$ of root length in untreated plants (Fig. $6 \mathrm{E}$ ). This indicates that RNH1C affects sensitivity to hydroxyurea and is likely to be involved in DNA replication.

\section{DISCUSSION}

RNase H1 proteins are known to digest the RNA component of RNA:DNA hybrids and this substrate must contain at least four sequential ribonucleotides ${ }^{16}$. In contrast, RNase $\mathrm{H} 2$ complexes only require a single ribonucleotide incorporated into double-stranded DNA for activity ${ }^{1,31}$. Therefore, RNase $\mathrm{H} 2$ has a broader substrate range and is partially redundant with RNase $\mathrm{H}^{32}$. We demonstrate for the first time that Arabidopsis thaliana RNH1A and RNH1B have the canonical RNase H1 activity. Because the presence of RNase H2 complexes has been previously shown ${ }^{2,3}$, this indicates that plants, like other eukaryotes, have both RNase $\mathrm{H} 1$ and $\mathrm{H} 2$. Our results suggest that all three RNase $\mathrm{H} 1$ genes identified in the Arabidopsis thaliana genome encode canonical bona fide RNase H1 proteins.

Arabidopsis thaliana contains four RNase H1 proteins. Their evolutionary origin may be traced down to three events. The first event occurred in the common ancestor of dicots, where nuclear and organellar paralogs have been formed. An independent event in the common ancestor of monocots (or possibly the common ancestor of all angiosperms) led to the formation of similar, yet evolutionarily independent nuclear and organellar paralogs in monocots. Nuclear and organellar RNases H1 do not display any obvious structural differences beyond the presence or absence of a transit peptide/presequence. Therefore, it is unknown if they are functionally distinct beyond having different subcellular localizations and expression patterns.

The second event leading to the formation of four RNase H1 proteins in Arabidopsis thaliana occurred in the common ancestor of Brassicaceae. The ancestral organellar protein diversified into two proteins, which are represented by RNH1B and RNH1C in Arabidopsis. Although these proteins are localized to mitochondria and chloroplasts ${ }^{23}$, their ortologs in Brassicaceae do not have consistent predicted localizations and the functional impact of this event remains unknown. The third event is alternative splicing of RNH1B, which results in translation of proteins predicted to localize to mitochondria or the nucleus. This mechanism is reminiscent of dual localization of metazoan RHases $\mathrm{H}^{22}$. Overall, our results are consistent with recent evidence that each genome within Arabidopsis thaliana cells has at least one RNase $\mathrm{H}^{23}$. However, the nuclear genome is served by two RNase H1 proteins. Additionally, most eukaryotes, including Arabidopsis thaliana contain RNase H2 complexes, which are nuclear localized and at least partially redundant with RNases $\mathrm{H} 1^{2,31,32}$. This may suggests 
that the nuclear genome requires the presence of several activities resolving RNA:DNA hybrids.

Our results indicate that at least one organellar RNase $\mathrm{H} 1$ is needed for proper embryonic development. This is consistent with data from metazoans, where RNase H1 is crucial for proper mitochondrial DNA replication during embryo development ${ }^{27,33,34}$. Viability of single atrnhlb and atrnhlc mutants may speculatively be explained by dual (mitochondrial and plastid) localization of those proteins, which is not an uncommon phenomenon ${ }^{35,36}$. Alternatively, defects in both mitochondria and chloroplasts may have a synergistic effect in embryonic development.

RNase H1 has been shown to be required for genome maintenance in chloroplasts ${ }^{23}$. Our observation that plastid DNA copy number is substantially increased in atrnh1c may indicate that genome instability in atrnhlc leads to overamplification of the entire genome. Because the plastid genome is copied by a combination of replication and recombination ${ }^{37}$, this overamplification may rely on homologous recombination. Interestingly, loss of mitochondrial RNH1B does not affect the level of mitochondrial DNA. This may suggest that, contrary to animals, plant mitochondria contain enzymes at least partially redundant with RNH1s.

The reported role of RNH1C in release of replication-transcription conflicts and chloroplast DNA integrity ${ }^{23}$ is consistent with our observations that atrnhlc mutation increases sensitivity to hydroxyurea. This may be interpreted as evidence of disrupted DNA replication in atrnhlc. This result should however be interpreted carefully because hydroxyurea inhibits synthesis of deoxyribonucleotides and increases misincorporation of ribonucleotides ${ }^{29,30}$, which may have a replication-independent effect on RNase H1-dependent processes. Additionally, hydroxyurea may cause oxidative stress ${ }^{38-40}$, which could also have a replication-independent effect.

Our results raise questions about the roles of nuclear and mitochondrial RNases H1 in Arabidopsis thaliana. Single mutations in genes encoding these proteins do not result in visible phenotypes, which may likely be attributed to protein redundancy and localization to multiple cellular compartments. Resolving the roles of those proteins remain an important goal for future studies.

\section{MATERIALS AND METHODS}

Plant material and oligonucleotides 
Plant lines used in this study: Col-0 (CS 70000), atrnh1a (SALK_150285C), atrnh1b

(SAIL_1174_C11), atrnh1c (SAIL_97_E11) and crosses between abovementioned.

Oligonucleotides used for expression analysis: P1: 5'-TTTAGTTTGGGTTGATGGGTTCC3' + 5'-CACACTCATTGCAGGATGTGATAC-3'; P2: 5'-

ATCTCTTAGACGGGGAAGATTTGT-3' + 5'-AATGCAAGTCATGTCAAAGATGAT-3'; P3: 5'-TCAATAATGCAAGTTTCATATGAGGT-3' + 5'-

GTTTGGAGCTCTTACACCTTGTCT-3'; P4: 5'-ATCCCTTATAAACGCTAACTGGAG3' + 5'-TCAAGTTGGATCTTCGGTTTATG-3'; P5: 5'-

TACACCATGTCTTTTCCAGGAG-3' + 5'-GATATAGAAGCTGAAGGAAGTTGATCT3'; P6: 5'-AAAGATCCGGAGTTACACACTAGC-3' + 5'-

CATTCTGGTTCTTCACCAGTTTCT-3'. Sequences of oligonucleotides used for organellar DNA content quantification were previously described by Kim et $\mathrm{al}^{41}$.

\section{Phylogenetic analysis}

Sequences of putative plant RNH1 proteins were retrieved from Phytozome ${ }^{42}, \mathrm{CoGE}^{43}$, Ensembl Plants ${ }^{44}, \mathrm{PLAZA}^{45}$ and $1 \mathrm{KP}^{46}$ through BLAST search and aligned in MAFFT ${ }^{47}$. Only sequences containing full length hybrid binding domain (HBD), RNASEH1-like domain and four amino acids (DEDD) crucial for catalytic activity ${ }^{17}$, were kept. BAli-Phy ${ }^{48}$ with default settings on CIPRES $^{49}$ platform was used for phylogenetic analysis. Consensus tree was generated with $25 \%$ burn-in and visualized using FigTree. Subcellular localization was predicted by TargetP $\mathrm{P}^{26}$.

\section{In vitro activity of RNH1s}

6xHis-tagged HBDs and catalytic domains of RNH1A and B were expressed in E. coli from pET28 plasmid. Tagged proteins were bound on HisTrap (HP)(GE Healthcare) and eluted with increasing imidazole concentration. Enzymatic reaction was performed in Reaction Buffer (60mM NaCl, 16mM HEPES-KOH pH=7.0, 4\% glycerol, 0.8mM DTT, $80 \mu \mathrm{g} / \mathrm{ml}$ BSA, $4 \mathrm{mM} \mathrm{MgCl} 2$ ) with $20 \mathrm{nM}$ fluor-labelled substrate and $2 \mathrm{nM}-2 \mu \mathrm{M}$ purified RNH1 in $20 \mu \mathrm{l}$ reaction. Reaction was conducted for $30 \mathrm{~min}$ at $37^{\circ} \mathrm{C}$ and stopped by addition of EDTA to final concentration of $40 \mathrm{mM}$. Products were analyzed on $15 \%$ denaturing TBE-urea polyacrylamide gels and visualized by fluorescence readout.

\section{Chlorophyll content measurement}


Chlorophyll content was determined as described by Lichtenthaler ${ }^{50}$. Briefly, $1 \mathrm{~g}$ of frozen 2-3 week old seedlings was ground in liquid nitrogen and chlorophyll was extracted with $100 \%$ acetone. Samples were centrifuged at $10000 \mathrm{x}$ g for $10 \mathrm{~min}$ at $4^{\circ} \mathrm{C}$ and absorbance was measured at 645 and $662 \mathrm{~nm}$. Chlorophyll concentration was calculated using following formula: $\mathrm{C}_{\mathrm{a}+\mathrm{b}}(\mu \mathrm{g} / \mathrm{ml})=18.09 \times \mathrm{A} 645+7.05 \times \mathrm{A} 662$.

\section{Microscopic observations}

Mature siliques of Col-0 and $r n h 1 b^{-/-} / r n h l c^{+/-}$plants were destained in $70 \%$ ethanol and photographed under preparative microscope. For embryos observations siliques of Col- 0 and $r n h 1 b^{-/-} / r n h 1 c^{+/-}$were dissected, seeds were split based on morphology and cleared for $2 \mathrm{~h}$ at room temperature with Visikol ${ }^{51}$. Embryos inside seeds were visualized using Nomarski optics.

\section{Organellar DNA content quantification and expression analysis}

Total DNA was isolated from 2-3 weeks old seedlings with DNeasy Plant Mini Kit (QIAGEN). 1ng of DNA per qPCR reaction was used as template. For expression analysis total RNA was extracted with RNeasy Plant Mini Kit (QIAGEN) from 2-3 weeks old seedlings and 500ng was treated with DNaseI (Ambion) and used for reverse transcription. $0.5 \mathrm{ng}$ of cDNA was used as template in qPCR. Significance was determined using confidence intervals.

\section{Replicative stress assay}

Seeds were germinated on $1 / 2$ MS plates supplemented with $1 \%$ sucrose and $0.75 \%$ agar. After 1 week seedlings were transferred to liquid $1 / 2 \mathrm{MS}+1 \%$ sucrose with 0 or $2 \mathrm{mM}$ hydroxyurea (HU) and grown in continuous light. After 2 weeks root length was measured.

\section{ACKNOWLEDGEMENTS}

We want to thank Steven Smith for his help with phylogenetic analysis as well as Karolina Górecka and Małgorzata Figiel for their help with protein purification and biochemical activity assays. This work was supported by the National Science Foundation grant MCB 1120271 and the National Science Centre, Poland grant Polonez 2015/19/P/NZ1/03619 to A.T.W. This project has received funding from the European Union's Horizon 2020 research and innovation programme under the Marie Skłodowska-Curie grant agreement No 665778. 


\section{FIGURE LEGENDS}

Figure 1. Evolutionary origin of plant RNase H1 proteins. Phylogenetic tree of all full length predicted RNase H1 proteins. Support values at tree branches are posterior probability scores, which integrate over the uncertainty in both the alignment and the phylogeny. Predicted protein localizations obtained using TargetP are marked with colors. Mitochondria - red, chloroplast - green, other - blue. A version of this phylogenetic tree with all species names and support values is shown in Fig. S1.

Figure 2. Origin of four RNase H1 proteins in Arabidopsis thaliana. (A) Predicted localization of RNase H1 proteins from distinct clades identified in Fig 1. (B) Detailed phylogenetic tree of all RNase H1 proteins in Brassicaceae. Support values and color-coded predicted localization like in Fig 1. (C) Splice variants of $R N H 1 B$ predicted in Araport11. (D) 5' RACE of RNHIB. Asterisk indicates a non-specific PCR product. (E) Domain composition of four RNase H1 proteins in Arabidopsis thaliana.

Figure 3. RNH1A and RNH1B proteins from Arabidopsis thaliana exhibit canonical RNase H1 activity in vitro. Recombinant RNH1A (A) and RNH1B (B) were incubated with labeled oligonucleotide substrates containing different combinations of deoxyribonucleotides (black) and ribonucleotides (red).

Figure 4. Effects of mutations in genes encoding RNase H1 proteins. Approximately 3-weekold plants of Col-0 wild type (A), atrnhla (B), atrnh1b (C), atrnhlc (D), atrnhla, atrnh1b double mutant (E), atrnhla, atrnhlc double mutant (F), atrnhla, atrnhlb double mutant heterozygous for atrnhlc (G) and atrnhla, atrnh1b, atrnhlc triple mutant expressing RNH1C::RNH1C-GFP (H). (I) Relative chlorophyll content of Col-0 wild type and mutants in genes encoding RNase H1 proteins. Error bars indicate standard deviation from three biological replicates. (J) Schematic representation of RNH1 genes in A. thaliana. Arrows indicate START codons, triangles indicate T-DNA insertion positions. Red boxes indicate positions of the conserved RNase domain. $(\mathbf{K}, \mathbf{L})$ Expression of genes encoding RNases H1 measured (K) upstream of T-DNA insertion and (L) downstream of T-DNA insertion. Primer pairs are marked in $(\mathrm{J})$. Error bars indicate standard deviation from three biological replicates. Asterisks indicate significant change in expression determined by $95 \%$ confidence intervals. 
Figure 5. At least one organellar RNase H1 is required for embryo development. (A) Mature silique of Col-0 wild type. (B) atrnhla, atrnh1b double mutant heterozygous for atrnh1c $\left(\right.$ atrnh $\left.1 \mathrm{a} / \mathrm{b} / \mathrm{c}^{+/}\right)$. (C) Percentage of properly developing seeds in siliques. Error bars indicate standard deviation from three biological replicates. (D) Development of a Col-0 wild-type embryo. (E) Development of an aborted embryo.

Figure 6. Loss of RNH1C leads to increased chloroplast DNA content and hypersensitivity to hydroxyurea. Relative levels of nuclear (A), mitochondrial (B) and chloroplast (C) DNA in Col-0 wild type atrnh1 mutants. Asterisks indicate significant changes with $\mathrm{p}<0.05$. (D) Phenotype of plants subjected to HU stress. (E) Relative root length of Col-0 wild type and atrnh1 mutants upon $2 \mathrm{mM}$ hydroxyurea treatment. Asterisks indicate $\mathrm{p}<0.0001$ determined using 2-way ANOVA.

\section{SUPPLEMENTAL DATA}

Figure S1. Evolutionary origin of plant RNase H1 proteins. Phylogenetic tree of all full length predicted RNase H1 proteins as shown in Fig. 1 with all species names and support values included.

File S1. Aminoacid sequences of all RNase H1 proteins shown in Fig. 1 and Fig. S1.

\section{REFERENCES}

1. Cerritelli, S. M. \& Crouch, R. J. Ribonuclease H: the enzymes in eukaryotes. FEBS J. 276, 1494-1505 (2009).

2. Kalhorzadeh, P. et al. Arabidopsis thaliana RNase H2 deficiency counteracts the needs for the WEE1 checkpoint kinase but triggers genome instability. Plant Cell 26, 3680-3692 (2014).

3. Eekhout, T., Kalhorzadeh, P. \& De Veylder, L. Lack of RNase H2 activity rescues HUsensitivity of WEE1 deficient plants. Plant Signal. Behav. 10, (2015).

4. Stein, H. \& Hausen, P. Enzyme from calf thymus degrading the RNA moiety of DNARNA Hybrids: effect on DNA-dependent RNA polymerase. Science 166, 393-395 (1969).

5. Lima, W. F. et al. Human RNase H1 discriminates between subtle variations in the structure of the heteroduplex substrate. Mol. Pharmacol. 71, 83-91 (2007).

6. Santos-Pereira, J. M. \& Aguilera, A. R loops: new modulators of genome dynamics and function. Nat. Rev. Genet. 16, 583-597 (2015). 
7. Boque-Sastre, R. et al. Head-to-head antisense transcription and R-loop formation promotes transcriptional activation. Proc. Natl. Acad. Sci. U. S. A. 112, 5785-5790 (2015).

8. Stuckey, R., García-Rodríguez, N., Aguilera, A. \& Wellinger, R. E. Role for RNA:DNA hybrids in origin-independent replication priming in a eukaryotic system. Proc. Natl. Acad. Sci. U. S. A. 112, 5779-5784 (2015).

9. Cloutier, S. C. et al. Regulated Formation of lncRNA-DNA Hybrids Enables Faster Transcriptional Induction and Environmental Adaptation. Mol. Cell 61, 393-404 (2016).

10. Stirling, P. C. \& Hieter, P. Canonical DNA Repair Pathways Influence R-Loop-Driven Genome Instability. J. Mol. Biol. 429, 3132-3138 (2017).

11. Hamperl, S. \& Cimprich, K. A. The contribution of co-transcriptional RNA:DNA hybrid structures to DNA damage and genome instability. DNA Repair 19, 84-94 (2014).

12. Sollier, J. et al. Transcription-coupled nucleotide excision repair factors promote Rloop-induced genome instability. Mol. Cell 56, 777-785 (2014).

13. Arora, R. et al. RNaseH1 regulates TERRA-telomeric DNA hybrids and telomere maintenance in ALT tumour cells. Nat. Commun. 5, 5220 (2014).

14. Yu, K., Chedin, F., Hsieh, C.-L., Wilson, T. E. \& Lieber, M. R. R-loops at immunoglobulin class switch regions in the chromosomes of stimulated B cells. Nat. Immunol. 4, 442-451 (2003).

15. Sun, Q., Csorba, T., Skourti-Stathaki, K., Proudfoot, N. J. \& Dean, C. R-loop stabilization represses antisense transcription at the Arabidopsis FLC locus. Science 340, 619-621 (2013).

16. Nowotny, M., Gaidamakov, S. A., Crouch, R. J. \& Yang, W. Crystal structures of RNase $\mathrm{H}$ bound to an RNA/DNA hybrid: substrate specificity and metal-dependent catalysis. Cell 121, 1005-1016 (2005).

17. Nowotny, M. et al. Structure of Human RNase H1 Complexed with an RNA/DNA Hybrid: Insight into HIV Reverse Transcription. Mol. Cell 28, 264-276 (2007).

18. Evans, S. P. \& Bycroft, M. NMR structure of the N-terminal domain of Saccharomyces cerevisiae RNase HI reveals a fold with a strong resemblance to the Nterminal domain of ribosomal protein L9. J. Mol. Biol. 291, 661-669 (1999).

19. Ohtani, N., Yanagawa, H., Tomita, M. \& Itaya, M. Identification of the first archaeal Type 1 RNase H gene from Halobacterium sp. NRC-1: archaeal RNase HI can cleave an RNA-DNA junction. Biochem. J. 381, 795-802 (2004). 
20. Ohtani, N., Yanagawa, H., Tomita, M. \& Itaya, M. Cleavage of double-stranded RNA by RNase HI from a thermoacidophilic archaeon, Sulfolobus tokodaii 7. Nucleic Acids Res. 32, 5809-5819 (2004).

21. Tadokoro, T. \& Kanaya, S. Ribonuclease H: molecular diversities, substrate binding domains, and catalytic mechanism of the prokaryotic enzymes. FEBS J. 276, 1482-1493 (2009).

22. Suzuki, Y. et al. An upstream open reading frame and the context of the two AUG codons affect the abundance of mitochondrial and nuclear RNase H1. Mol. Cell. Biol. 30, 5123-5134 (2010).

23. Yang, Z. et al. RNase H1 Cooperates with DNA Gyrases to Restrict R-Loops and Maintain Genome Integrity in Arabidopsis Chloroplasts. Plant Cell 29, 2478-2497 (2017).

24. $\mathrm{Xu}, \mathrm{W}$. et al. The $\mathrm{R}-$ loop is a common chromatin feature of the Arabidopsis genome. Nat. Plants 3, 704-714 (2017).

25. Suchard, M. A. \& Redelings, B. D. BAli-Phy: simultaneous Bayesian inference of alignment and phylogeny. Bioinforma. Oxf. Engl. 22, 2047-2048 (2006).

26. Emanuelsson, O., Nielsen, H., Brunak, S. \& von Heijne, G. Predicting subcellular localization of proteins based on their N-terminal amino acid sequence. J. Mol. Biol. 300, 1005-1016 (2000).

27. Cerritelli, S. M. et al. Failure to produce mitochondrial DNA results in embryonic lethality in Rnaseh1 null mice. Mol. Cell 11, 807-815 (2003).

28. Cheng, C.-Y. et al. Araport11: a complete reannotation of the Arabidopsis thaliana reference genome. Plant J. Cell Mol. Biol. 89, 789-804 (2017).

29. Arudchandran, A. et al. The absence of ribonuclease $\mathrm{H} 1$ or $\mathrm{H} 2$ alters the sensitivity of Saccharomyces cerevisiae to hydroxyurea, caffeine and ethyl methanesulphonate: implications for roles of RNases H in DNA replication and repair. Genes Cells Devoted Mol. Cell. Mech. 5, 789-802 (2000).

30. Yarbro, J. W. Mechanism of action of hydroxyurea. Semin. Oncol. 19, 1-10 (1992).

31. Chon, H. et al. RNase $\mathrm{H} 2$ roles in genome integrity revealed by unlinking its activities. Nucleic Acids Res. 41, 3130-3143 (2013).

32. Zimmer, A. D. \& Koshland, D. Differential roles of the RNases H in preventing chromosome instability. Proc. Natl. Acad. Sci. U. S. A. 113, 12220-12225 (2016).

33. Holmes, J. B. et al. Primer retention owing to the absence of RNase H1 is catastrophic for mitochondrial DNA replication. Proc. Natl. Acad. Sci. U. S. A. 112, 9334-9339 (2015). 
34. Lima, W. F. et al. Viable RNaseH1 knockout mice show RNaseH1 is essential for R loop processing, mitochondrial and liver function. Nucleic Acids Res. 44, 5299-5312 (2016).

35. Carrie, C., Giraud, E. \& Whelan, J. Protein transport in organelles: Dual targeting of proteins to mitochondria and chloroplasts. FEBS J. 276, 1187-1195 (2009).

36. Silva-Filho, M. C. One ticket for multiple destinations: dual targeting of proteins to distinct subcellular locations. Curr. Opin. Plant Biol. 6, 589-595 (2003).

37. Oldenburg, D. J. \& Bendich, A. J. DNA maintenance in plastids and mitochondria of plants. Front. Plant Sci. 6, 883 (2015).

38. Wood, K. C. \& Granger, D. N. Sickle Cell Disease: Role of Reactive Oxygen and Nitrogen Metabolites. Clin. Exp. Pharmacol. Physiol. 34, 926-932

39. Burkitt, M. J. Nitric oxide generation from hydroxyurea: significance and implications for leukemogenesis in the management of myeloproliferative disorders. Blood 107, 2219$2222(2006)$.

40. Sakano, K., Oikawa, S., Hasegawa, K. \& Kawanishi, S. Hydroxyurea induces sitespecific DNA damage via formation of hydrogen peroxide and nitric oxide. Jpn. J. Cancer Res. Gann 92, 1166-1174 (2001).

41. Kim, M., Lee, U., Small, I., des Francs-Small, C. C. \& Vierling, E. Mutations in an Arabidopsis Mitochondrial Transcription Termination Factor-Related Protein Enhance Thermotolerance in the Absence of the Major Molecular Chaperone HSP101[W]. Plant Cell 24, 3349-3365 (2012).

42. Goodstein, D. M. et al. Phytozome: a comparative platform for green plant genomics. Nucleic Acids Res. 40, D1178-D1186 (2012).

43. Lyons, E. \& Freeling, M. How to usefully compare homologous plant genes and chromosomes as DNA sequences. Plant J. Cell Mol. Biol. 53, 661-673 (2008).

44. Kersey, P. J. et al. Ensembl Genomes 2018: an integrated omics infrastructure for nonvertebrate species. Nucleic Acids Res. 46, D802-D808 (2018).

45. Van Bel, M. et al. PLAZA 4.0: an integrative resource for functional, evolutionary and comparative plant genomics. Nucleic Acids Res. 46, D1190-D1196 (2018).

46. Matasci, N. et al. Data access for the 1,000 Plants (1KP) project. GigaScience 3, 17 (2014).

47. Katoh, K., Kuma, K., Toh, H. \& Miyata, T. MAFFT version 5: improvement in accuracy of multiple sequence alignment. Nucleic Acids Res. 33, 511-518 (2005). 
48. Suchard, M. A. \& Redelings, B. D. BAli-Phy: simultaneous Bayesian inference of alignment and phylogeny. Bioinforma. Oxf. Engl. 22, 2047-2048 (2006).

49. Miller, M. A., Pfeiffer, W. \& Schwartz, T. Creating the CIPRES Science Gateway for inference of large phylogenetic trees. in 2010 Gateway Computing Environments Workshop (GCE) 1-8 (2010). doi:10.1109/GCE.2010.5676129

50. Lichtenthaler, H. K. [34] Chlorophylls and carotenoids: Pigments of photosynthetic biomembranes. in Methods in Enzymology 148, 350-382 (Academic Press, 1987).

51. Villani, T. S., Koroch, A. R. \& Simon, J. E. An improved clearing and mounting solution to replace chloral hydrate in microscopic applications1. Appl. Plant Sci. 1, (2013). 
Figure 1

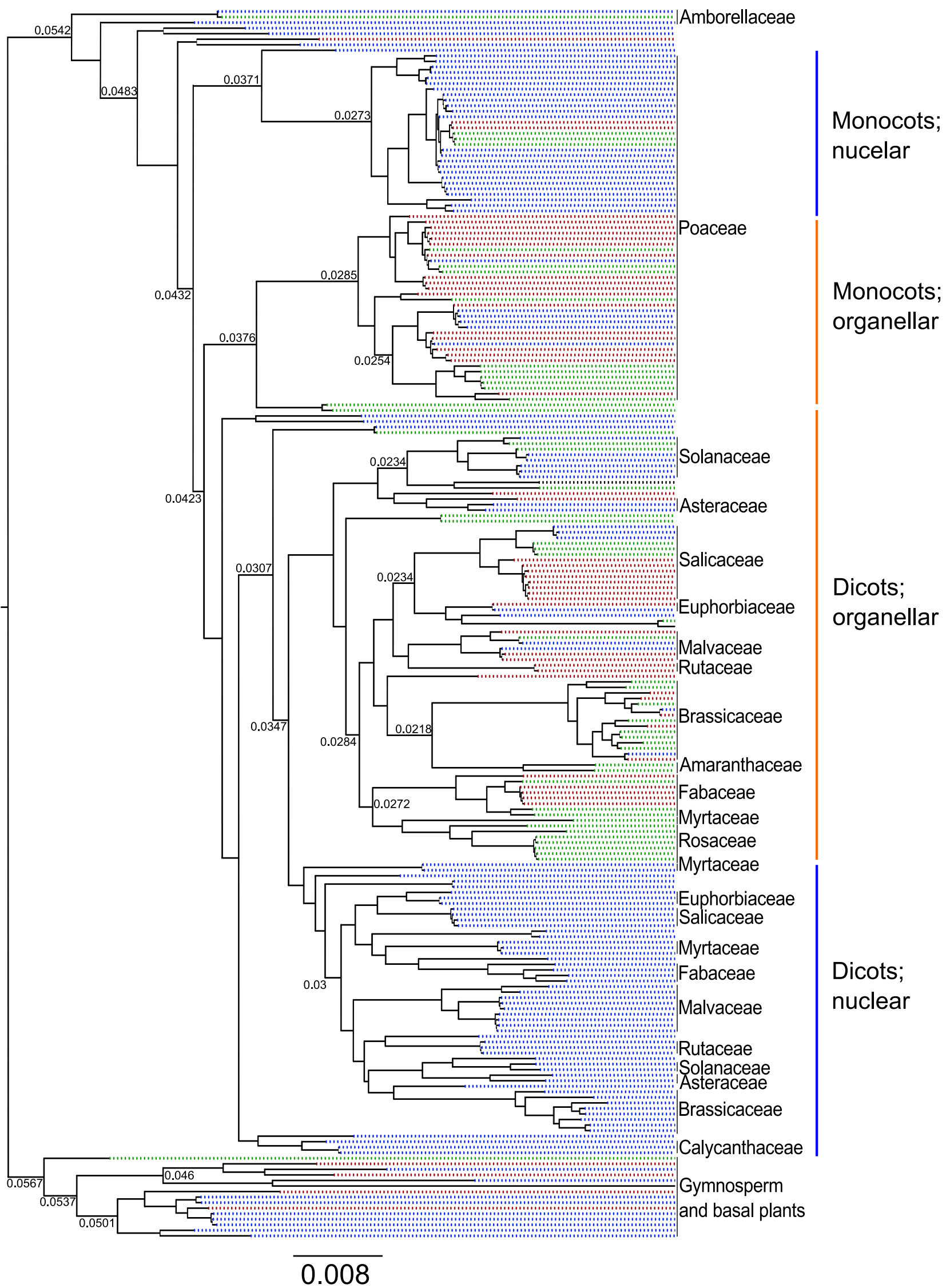


Figure 2

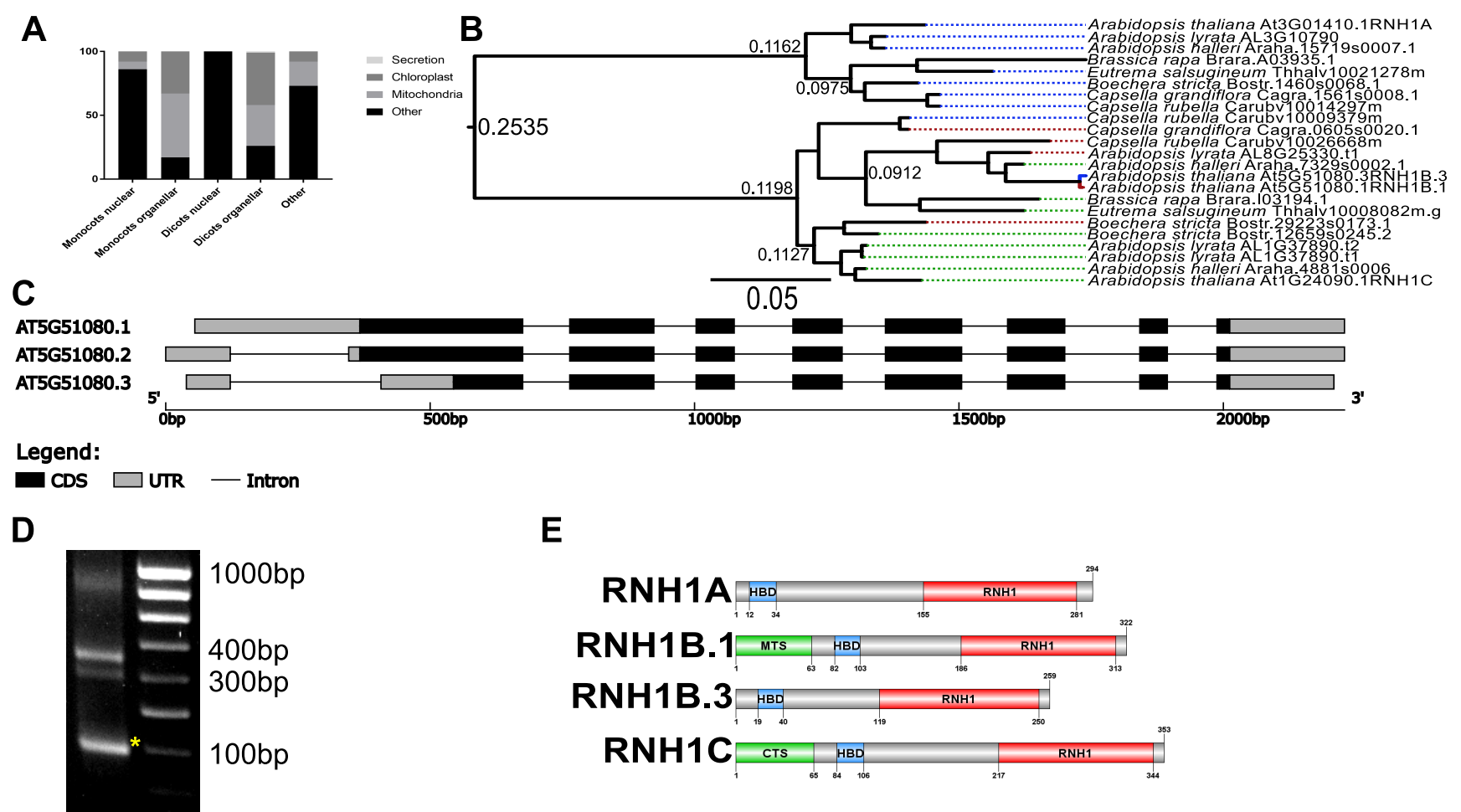


bioRxiv preprint doi: https://doi.org/10.1101/662080; this version posted June 23, 2020. The copyright holder for this preprint (which was not certified by peer review) is the author/funder. All rights reserved. No reuse allowed without permission.

\section{Figure 3}

A

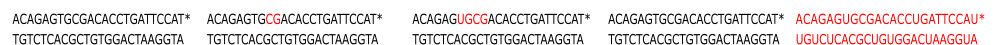
TGTCTACGCTGTGGACTAAGG

Ratio
Enzyme.substat

Buffer 1:100 1:10 1:1 10:1 Buffer 1:100 1:10 1:1 10:1 Buffer 1:100 1:10 1:1 10:1

- - - - - -

$-\infty$

B

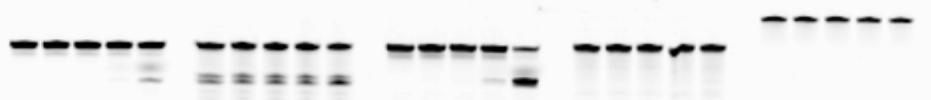


Figure 4

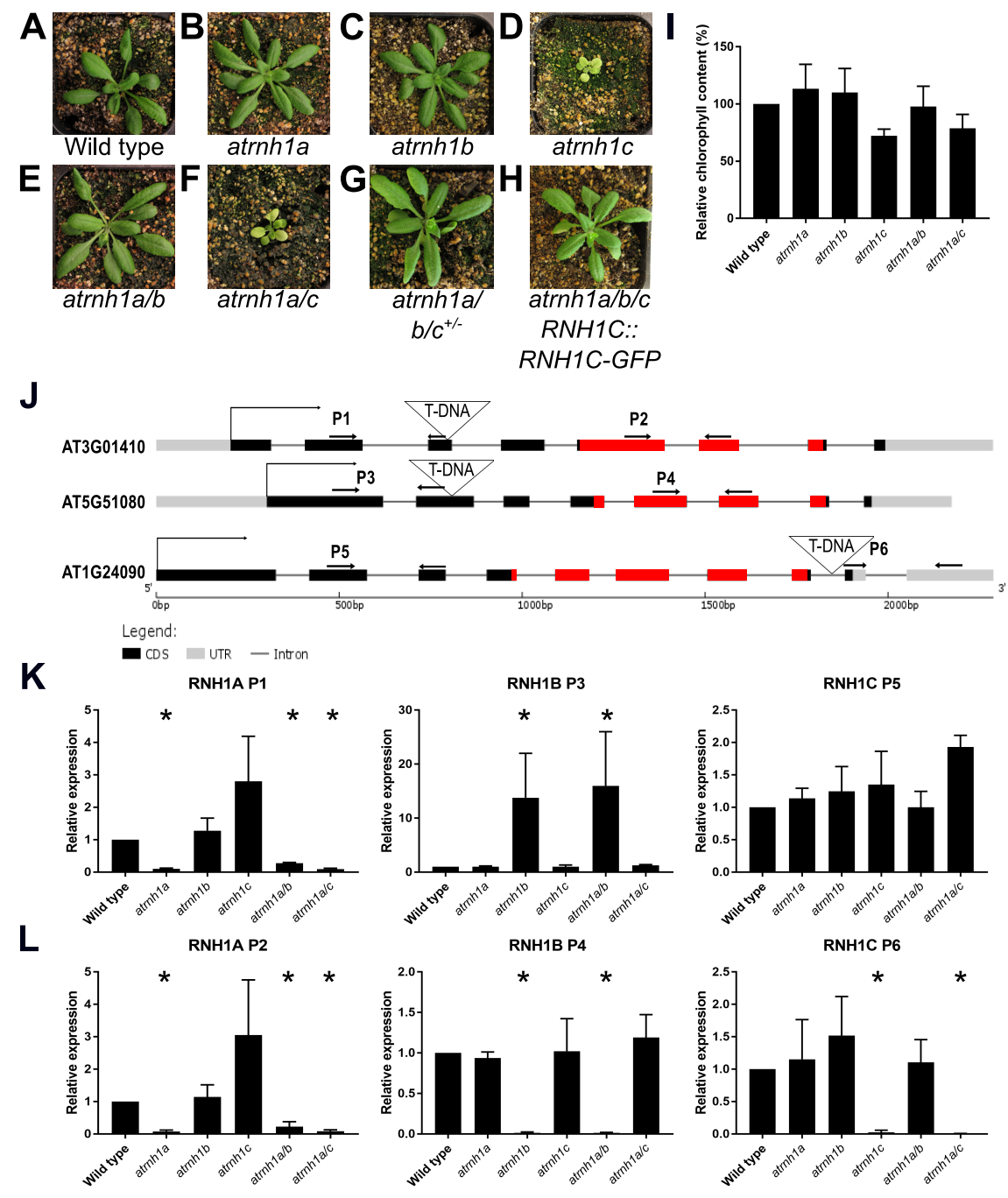




\section{Figure 5}

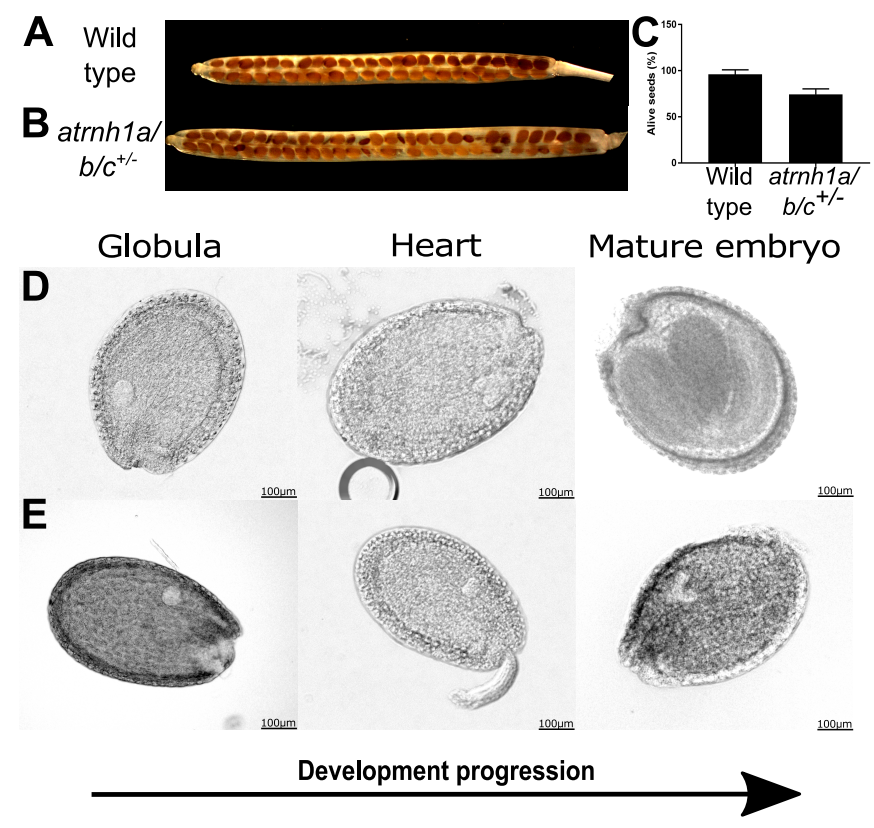




\section{Figure 6}
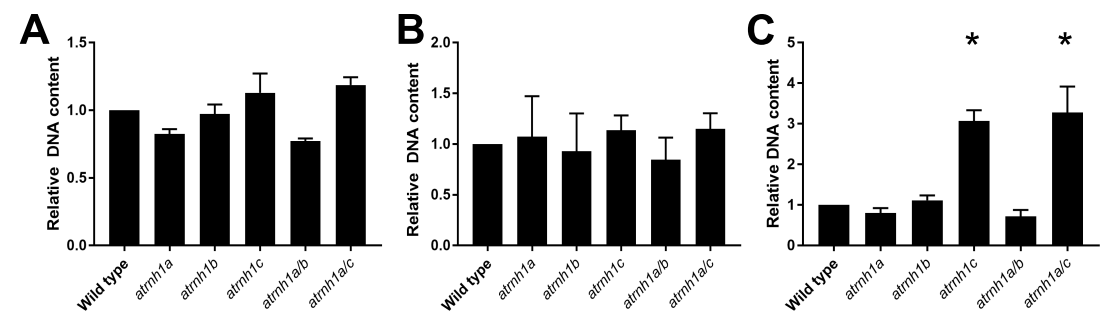

D

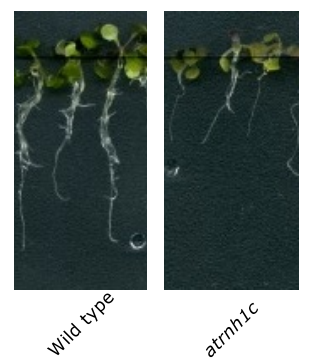

E

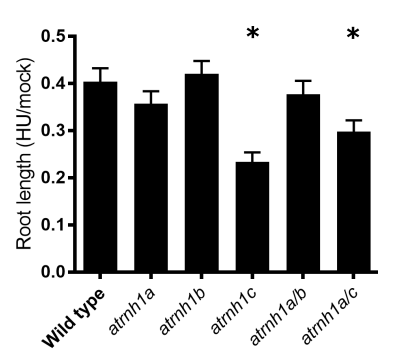




\section{Supplemental Figure 1}

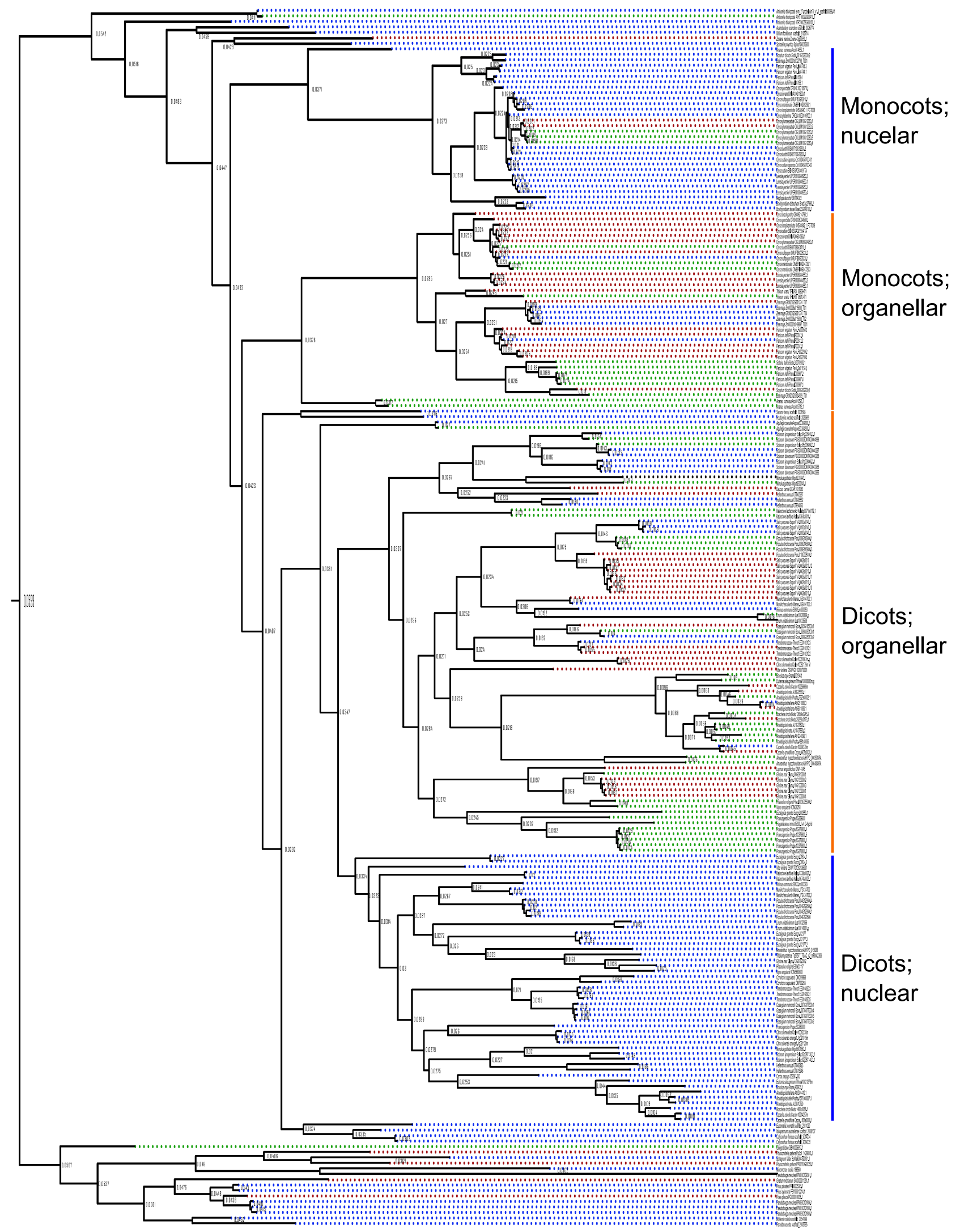

